Document downloaded from:

http://hdl.handle.net/10251/160592

This paper must be cited as:

Calabuig, JM.; Fernández-Unzueta, M.; Galaz-Fontes, F.; Sánchez Pérez, EA. (2019).

Completability and optimal factorization norms in tensor products of Banach function spaces. Revista de la Real Academia de Ciencias Exactas Físicas y Naturales Serie A Matemáticas. 113(4):3513-3530. https://doi.org/10.1007/s13398-019-00711-7

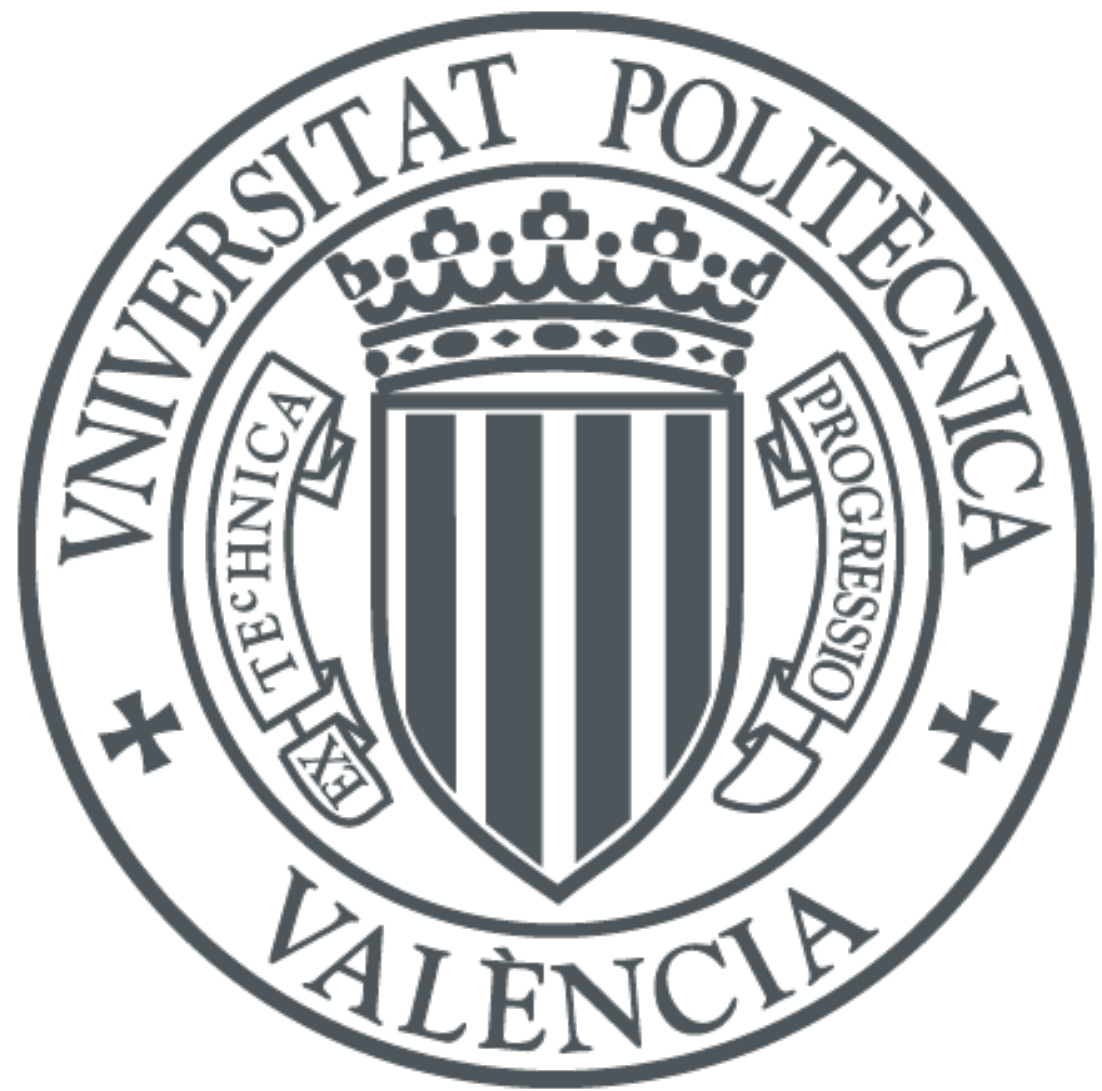

The final publication is available at

https://doi.org/10.1007/s13398-019-00711-7

Copyright Springer-Verlag

Additional Information 


\title{
COMPLETABILITY AND OPTIMAL FACTORIZATION NORMS IN TENSOR PRODUCTS OF BANACH FUNCTION SPACES
}

\author{
J.M. CALABUIG, M. FERNÁNDEZ-UNZUETA, F. GALAZ-FONTES, \\ AND E.A. SÁNCHEZ-PÉREZ
}

\begin{abstract}
Given $\sigma$-finite measure spaces $\left(\Omega_{1}, \Sigma_{1}, \mu_{1}\right)$ and $\left(\Omega_{2}, \Sigma_{2}, \mu_{2}\right)$, we consider Banach spaces $X_{1}\left(\mu_{1}\right)$ and $X_{2}\left(\mu_{2}\right)$, consisting of $L^{0}\left(\mu_{1}\right)$ and $L^{0}\left(\mu_{2}\right)$ measurable functions respectively, and study when the completion of the simple tensors in the projective tensor product $X_{1}\left(\mu_{1}\right) \otimes_{\pi} X_{2}\left(\mu_{2}\right)$ is continuously included in the metric space of measurable functions $L^{0}\left(\mu_{1} \otimes \mu_{2}\right)$. In particular, we prove that the elements of the completion of the projective tensor product of $L^{p}$-spaces are measurable functions with respect to the product measure. Assuming certain conditions, we finally show that given a bounded linear operator $T: X_{1}\left(\mu_{1}\right) \otimes_{\pi} X_{2}\left(\mu_{2}\right) \rightarrow E$ (where $E$ is a Banach space), a norm can be found for $T$ to be bounded, which is 'minimal' with respect to a given property -2 -rectangularity - The same technique may work for the case of $n$-spaces.
\end{abstract}

\section{INTRODUCTION}

Unfortunately, tensor products do not fit well with the Banach lattice structure. From the very beginning of the theory of topological tensor products, it was known that the projective tensor product of Banach lattices is not necessarily a Banach lattice. In fact, it is well-known that even the relevant space $\ell^{2} \widehat{\otimes}_{\pi} \ell^{2}$-where $\pi$ is the projective tensor norm-, is not a Banach lattice. In general, this is a problem for extending some relevant results for linear operators on Banach lattices to the bilinear setting.

Some efforts have been made to avoid this problem. For example, the lattice tensor product — developed by Fremlin and other authors, see [3, 4, 11, 12, 19]-, has sometimes played the role of the projective tensor product successfully when function lattices are involved. However, sometimes it cannot be used, for example when we are searching for optimal factorizations of bilinear maps.

Date: October 11, 2018.

2010 Mathematics Subject Classification. 46E30, 28A35, 47H60.

Key words and phrases. Product measure, Banach function space, bilinear operator, tensor product, factorization.

J.M. Calabuig and M. Fernández-Unzueta were supported by Ministerio de Economía, Industria y Competitividad (Spain) under project MTM2014-53009-P. M. Fernández-Unzueta was also suported by CONACyT 284110. F. Galaz-Fontes was supported by Ministerio de Ciencia e Innovación (Spain) and FEDER under project MTM2009-14483-C02-01. E.A. Sánchez Pérez was supported by Ministerio de Economía, Industria y Competitividad (Spain) under project MTM2016-77054-C2-1-P. 
Motivated in part by the so called optimal factorization of linear continuous operators acting on Banach function spaces [18], some efforts have been recently made for extending the characterization of the minimal factorization norm to the bilinear/multilinear case (see for example [5] and the references therein). The main problem in this case is that the optimal factorization one may expect to find, is not in general a Banach function space over the product measure. However, it will satisfy some weaker lattice-type properties. In this paper we characterize when the factorization space is at least a space of measurable functions with respect to the product measure. Thus, the interest is not only focused on the factorization problem, but also on the general structure of the tensor products of Banach function spaces. In order to do that, we first analyze a class of normed spaces of measurable functions with respect to the product measure endowed with some weak function lattice properties.

Let $(\Omega, \Sigma, \mu)$ be a $\sigma$-finite measure space and denote by $L^{0}(\mu)$ the space consisting of classes of $\Sigma$-measurable functions, where $f, g$ are identified if $f=g \mu$-a.e.. Then $L^{0}(\mu)$ can be given a metric so that it becomes a complete metric space. In this case, a Banach space $X \subset L^{0}(\mu)$ is continuously included if, and only if, $X$ has the subsequence property. This means that if $\left\{f_{n}\right\} \subset X$ is convergent to $f \in X$, then it has a subsequence that converges to $f \mu$-a.e. This is very well known when $\mu$ is a finite measure. In the $\sigma$-finite case, it seems this is not so and thus we present proofs for the corresponding results. Given a normed space $X \subset L^{0}(\mu)$ we give a necessary and sufficient condition for its completion $\widehat{X}$ to be continuously included in $L^{0}(\mu)$. We say that this kind of spaces are $L^{0}(\mu)$-completable.

Let $\left(\Omega_{1}, \Sigma_{1}, \mu_{1}\right)$ and $\left(\Omega_{2}, \Sigma_{2}, \mu_{2}\right)$ be $\sigma$-finite measure spaces and consider its product measure space $\left(\Omega_{1} \times \Omega_{2}, \Sigma_{1} \otimes \Sigma_{2}, \mu_{1} \otimes \mu_{2}\right)$. Given Banach function spaces $X_{1}\left(\mu_{1}\right)$ and $X_{2}\left(\mu_{2}\right)$, it is well known that, in general, the projective tensor product space $X_{1}\left(\mu_{1}\right) \widehat{\otimes}_{\pi} X_{2}\left(\mu_{2}\right)$ is not a lattice Banach space. However $X_{1}\left(\mu_{1}\right) \otimes_{\pi} X_{2}\left(\mu_{2}\right)$ possesses a relevant property which we have called 2-rectangularity. This indicates that if $A \in \Sigma_{1}, B \in \Sigma_{2}$ and $R$ is the 'rectangle' $A \times B$, then $\chi_{R} f \in X_{1}\left(\mu_{1}\right) \otimes X_{2}\left(\mu_{2}\right)$ and $\pi\left(\chi_{R} f\right) \leq C \pi(f), \forall f \in X_{1}\left(\mu_{1}\right) \otimes X_{2}\left(\mu_{2}\right)$, for some $C>0$.

We discuss two questions that arise in the situation we just described. First, we show that it is possible that the completion $X_{1}\left(\mu_{1}\right) \widehat{\otimes}_{\pi} X_{2}\left(\mu_{2}\right)$ be continuously included in $L^{0}\left(\mu_{1} \otimes \mu_{2}\right)$. This is clear for example if $X_{1}\left(\mu_{1}\right)=L^{1}\left(\mu_{1}\right)$ and $X_{1}\left(\mu_{2}\right)=$ $X_{2}\left(\mu_{2}\right)$, since in this case $L^{1}(\mu) \widehat{\otimes}_{\pi} L^{1}\left(\mu_{2}\right)=L^{1}\left(\mu_{1} \otimes \mu_{2}\right)$. However, to our knowledge the general question has not been considered before for a non-atomic measure. Specifically we establish that $L^{p}\left(\mu_{1}\right) \widehat{\otimes}_{\pi} L^{p}\left(\mu_{2}\right)$ is continuously included in $L^{0}\left(\mu_{1} \otimes\right.$ $\mu_{2}$ ) when $1<p<\infty$. Take $\Omega_{1}=\mathbb{N}=\Omega_{2}$ and let $\mu_{1}$ and $\mu_{2}$ be the corresponding counting measure. Then we show that $\ell^{p} \widehat{\otimes}_{\pi} \ell^{r}, 1 \leq p, r<\infty$ is continuously included in $\ell^{\infty}\left(\mathbb{N}^{2}\right)$, the set of bounded functions defined on $\mathbb{N}^{2}$. This was already noticed before in [14], although no proof was given there. Some other related results can also be found in [13, p. 1364]. The main property on which these results are based is that, under mild conditions on the measure $\mu$, the space $L^{p}(\mu)$ has a Schauder basis if $1 \leq p<\infty$. 
The other question we discuss, and with which we end our work, is that of given a bounded linear operator $T: X_{1}\left(\mu_{1}\right) \otimes_{q} X_{2}\left(\mu_{2}\right) \rightarrow E$, where $E$ is a Banach space, to find a norm on $X_{1}\left(\mu_{1}\right) \otimes X_{2}\left(\mu_{2}\right)$ that is minimal with respect to the condition of 2-rectangularity and boundedness of $T$.

Finally, let us note that the same arguments should work for the case in which $n$ spaces are involved.

\section{NOTATION AND PRELIMINARIES}

2.1. Some general notation. In this work we will only consider real vector spaces. When $X$ is a normed space, then $B_{X}:=\{x \in X:|| x \mid \leq 1\}$ and $X^{*}$ is the normed dual space of $X$. If convenient, the norm in $X$ will also be indicated as $\|\cdot\|_{X}$. Let $M$ be a metric space. Given a sequence $\left\{f_{n}\right\} \subset M$ and $f \in M$, to express that $\left\{f_{n}\right\}$ converges to $f$ we write $f_{n} \stackrel{M}{\rightarrow} f$. In all cases $\Omega$ is a non-empty set and $2^{\Omega}:=\{A: A \subset \Omega\}$. By $F(\Omega)$ we represent the vector space consisting of all functions $f: \Omega \rightarrow \mathbb{R}$ and $X(\Omega)$ indicates that $X(\Omega)$ is a vector subspace of $F(\Omega)$. When we write $X=X(\Omega)$ we mean that the subspace $X(\Omega)$ will frequently be denoted simply by $X$.

2.2. Spaces of measurable functions. Throughout this work $(\Omega, \Sigma, \mu)$ is always a $\sigma$-finite measure space. Then $L^{0}(\mu)$ denotes the vector space consisting of all $\Sigma$-measurable functions, two of them being identified if they are equal $\mu$-almost everywhere ( $\mu$-a.e.). When $\mu(\Omega)<\infty$, then $L^{0}(\mu)$ is a complete metric space with the distance $d$ defined as

$$
d(f, g):=\int_{\Omega} \frac{|f-g|}{1+|f-g|} d \mu, \forall f, g \in L^{0}(\mu) .
$$

Note this distance is translation invariant and the following holds ([1, Thm. 1.82], [18, Sect. 2.1]).

Lemma 2.1. Let $\mu(\Omega)<\infty,\left\{f_{n}\right\} \subset L^{0}(\mu)$ and $f \in L^{0}(\mu)$. Then the following properties are equivalent:

i) $f_{n} \stackrel{L^{0}(\mu)}{\longrightarrow} f$.

ii) $\left\{f_{n}\right\}$ converges to $f$ in measure.

iii) Any subsequence of $\left\{f_{n}\right\}$ has a subsequence that converges pointwise to $f \mu$-a.e..

The notation $M(\mu)$ indicates that $M(\mu) \subset L^{0}(\mu)$ and in this case we will say that $M(\mu)$ is a space of measurable functions. By $M=M(\mu)$ we mean that the space $M(\mu)$ will frequently be denoted just by $M$. A metric space $M=M(\mu)$ satisfies the $\mu$-subsequence property (or simply the subsequence property) if any sequence $\left\{f_{n}\right\} \subset M$ that converges to $f \in M$ has a subsequence that converges pointwise to $f \mu$-a.e.. The following result is well known and can be obtained from Lemma 2.1.

Proposition 2.2. Let $\mu(\Omega)<\infty$. A metric space $M(\mu)$ has the subsequence property if, and only if, $M(\mu)$ is continuously included in $L^{0}(\mu)$. In particular, $L^{0}(\mu)$ has the subsequence property. 
2.3. Banach and Köthe function spaces. Let $(\Omega, \Sigma, \mu)$ be a $\sigma$-finite measure space. A Banach space $X=X(\mu)$ is a Banach function space (B.f.s.), with respect to $\mu$, if it is an ideal in $L^{0}(\mu)$ and has a Riesz norm. This means that if $0 \leq|g| \leq|f|$, where $g \in L^{0}(\mu)$ and $f \in X$, we always have that $g \in X$ and $\|g\| \leq\|f\|([16$, Sect.1.9], [20, Sects. 6.30, 15.63]). Here, by $f \leq g$ we understand that $f \leq g$ pointwise $\mu$-a.e. and we call this order the natural $\mu$-a.e. order. Notice that in this way a B.f.s. $X(\mu)$, endowed with the natural $\mu$-a.e. order, is a Banach lattice $[16$, Sect.2.11]. Hence any B.f.s. has the subsequence property [21, Thm.100.6].

Let $X_{1}=X_{1}\left(\mu_{1}\right)$ and $X_{2}=X_{2}\left(\mu_{2}\right)$ be B.f.s.. A linear operator $T: X_{1} \rightarrow X_{2}$ is positive if $T f \geq 0$ when $f \in X$ and $f \geq 0$. A positive linear operator between B.f.s. is always bounded [1, Lemma 3.22]. Assume that $X_{1} \subset X_{2}$. Since the inclusion $j$ from $X_{1}$ into $X_{2}$ is a positive linear map, it follows that an inclusion between B.f.s. is always bounded.

A normed space $X=X(\mu)$ is a 1-rectangular normed space if there is some real number $C>0$ such that whenever $f \in X$ and $A \in \Sigma$ we have $\chi_{A} f \in X$ and $\left\|\chi_{A} f\right\|_{X} \leq C\|f\|_{X}$. In this situation, the norm will also be called 1-rectangular and we will say that $C$ is a 1-rectangularity constant for $Z[6]$. When $X=X(\mu)$ is a Banach function space, note that $X$ is 1-rectangular, with 1 as a constant of rectangularity.

As usual, $L^{\infty}(\mu)$ is the B.f.s. of all $\mu$-essentially bounded functions $f: \Omega \rightarrow \mathbb{R}$ endowed with its natural norm and, for $1 \leq p<\infty, L^{p}(\mu)$ is the B.f.s. consisting of $p$-integrable functions $f: \Omega \rightarrow \mathbb{R}$ with the corresponding norm. When $X=X(\mu)$ is a B.f.s. and $f \chi_{A} \in L^{1}(\mu), \forall f \in X, \mu(A)<\infty$, we will say that $X$ is a B.f.s. of locally integrable functions. If additionally we have that $\chi_{A} \in X$ when $\mu(A)<\infty$, then the B.f.s. $X$ will be called Köthe function space (K.f.s.) ([15, Def 1.b.17], [2, Def. I.1.3]). Notice that for each $p \geq 1, L^{p}(\mu)$ is a K.f.s..

2.4. Product measure. A semiring in $\Omega$ is a collection $\mathcal{S} \subset 2^{\Omega}$ such that $\emptyset \in \mathcal{S}$ and when $A, B \in \mathcal{S}$, then $A \cap B \in \mathcal{S}$ and $A \backslash B$ is a finite union of disjoint sets in $\mathcal{S}$. If additionally $\Omega \in \mathcal{S}$, then a semiring is called semialgebra. If $\mathcal{S}_{1}$ is a semiring in $\Omega_{1}$ and $\mathcal{S}_{2}$ is a semiring in $\Omega_{2}$, then the collection $\mathcal{S}_{1} \widetilde{\times} \mathcal{S}_{2}:=\left\{A \times B: A \in \mathcal{S}_{1}, B \in \mathcal{S}_{2}\right\}$ is a semiring in $\Omega_{1} \times \Omega_{2}$. Let $\left(\Omega_{1}, \Sigma_{1}, \mu_{1}\right)$ and $\left(\Omega_{2}, \Sigma_{2}, \mu_{2}\right)$ be measure spaces. Then $\mu_{1} \otimes \mu_{2}$ is the product measure, defined on $\Sigma_{1} \otimes \Sigma_{2}$, the $\sigma$-algebra in $\Omega_{1} \times \Omega_{2}$ generated by the semiring of "rectangles" $\Sigma_{1} \tilde{\times} \Sigma_{2}$.

2.5. Normed tensor products. Given any two vector spaces $X_{1}$ and $X_{2}$, recall that its algebraic tensor product $X_{1} \otimes X_{2}$ is a vector space having the fundamental property that any bilinear map $B: X_{1} \times X_{2} \rightarrow E$, where $E$ is another vector space, can be factorized by a linear operator $T_{B}: X_{1} \otimes X_{2} \rightarrow E$ as shown below:

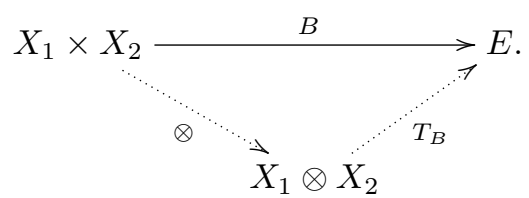


Let $j=1,2$ and $X_{j}, Y_{i}$ be vector spaces, and assume that $S: X_{1} \rightarrow Y_{1}$ and $T: X_{2} \rightarrow Y_{2}$ are linear operators. Then there is a unique linear map $S \otimes T:$ $X_{1} \otimes X_{2} \rightarrow Y_{1} \otimes Y_{2}$ satisfying

$$
S \otimes T(x \otimes y)=S x \otimes T y, \forall x \in X_{1}, y \in X_{2} .
$$

Let $X$ and $Y$ be normed spaces. Then on $X \otimes Y$ we have the projective norm

$$
\pi(t):=\inf \left\{\sum_{n=1}^{N}\left\|x_{n}\right\| \cdot\left\|y_{n}\right\|: x_{n} \in X, y_{n} \in Y, 1 \leq n \leq N, t=\sum_{n=1}^{N} x_{n} \otimes y_{n}\right\},
$$

and the injective norm

$$
\epsilon(t):=\sup \left\{\left|x^{*} \otimes y^{*}(t)\right|: x^{*} \in B_{X^{*}}, y^{*} \in B_{Y^{*}}\right\}, \forall t \in X \otimes Y .
$$

From now on, if $q$ is a norm on the tensor product $X \otimes Y$ the corresponding normed space will be indicated by $X \otimes_{q} Y$ and its completion by $X \widehat{\otimes}_{q} Y$.

Definition 2.3. Let $X$ and $Y$ be normed spaces.

a) A norm $q$ on $X \otimes Y$ is a reasonable crossnorm if:

i) $q(x \otimes y) \leq\|x\|\|y\|, \forall x \in X, y \in Y$.

ii) When $x^{*} \in X^{*}, y^{*} \in Y^{*}$, then $x^{*} \otimes y^{*} \in\left(X \otimes_{q} Y\right)^{*}$ and $\left\|x^{*} \otimes y^{*}\right\| \leq\left\|x^{*}\right\|\left\|y^{*}\right\|$.

b) A norm $q$ on $X \otimes Y$ is uniform if when $S: X \rightarrow X$ and $T: Y \rightarrow Y$ are bounded linear operators, then the linear operator $S \otimes T: X \otimes_{q} Y \rightarrow X \otimes_{q} Y$ is also bounded and $\|S \otimes T\| \leq\|S\|\|T\|$.

For any pair of normed spaces $X$ and $Y$, both the projective norm and the injective norm are uniform and reasonable crossnorms. The projective norm has additionally the universal property that if $E$ is a normed space and $B: X \times Y \rightarrow E$ is a bounded bilinear map, then the linear map appearing in $(2.2)$ is bounded.

Let $\left(\Omega_{j}, \Sigma_{j}, \mu_{j}\right)$ be a measure space for $j=1,2$, and take $\Omega=\Omega_{1} \times \Omega_{2}$, $\Sigma=\Sigma_{1} \otimes \Sigma_{2}, \mu=\mu_{1} \otimes \mu_{2}$. When $X_{1}=X_{1}\left(\mu_{1}\right)$ and $X_{2}=X_{2}\left(\mu_{2}\right)$ the tensor product $X_{1} \otimes X_{2}$ can be represented in more familiar terms as follows. Given $f_{1} \in X_{1}, f_{2} \in X_{2}$, let $f_{1} \times f_{2}$ temporarily denote the function defined on $\Omega$ by $f_{1} \times f_{2}(x, y):=f_{1}(x) f_{2}(y)$. Since $f_{1}$ is $\Sigma_{1}$-measurable and $f_{2}$ is $\Sigma_{2}$-mesurable, note that $f_{1} \times f_{2}$ is a $\Sigma$-measurable function. Take $f_{1}, g_{1}: \Omega_{1} \rightarrow \mathbb{R}$ and $f_{2}, g_{2}: \Omega_{2} \rightarrow \mathbb{R}$. If $f_{1}=g_{1} \mu_{1}$-a.e. and $f_{2}=g_{2} \mu_{2}$-a.e., we also have $f_{1} \times f_{2}=g_{1} \times g_{2} \mu$-a.e. This shows the correspondence $B: X_{1} \times X_{2} \rightarrow L^{0}(\mu)$ given by $B\left(f_{1}, f_{2}\right)=f_{1} \times f_{2}$ is well defined. Since $B$ is a bilinear map, the universal property of the algebraic tensor product $X \otimes Y$ indicates there is a linear map $J: X \otimes Y \rightarrow L^{0}(\mu)$ such that $J(f \otimes g)=f \times g, \forall f \in X, g \in Y$. It turns out that this map is injective and this allows us to identify $X \otimes Y$ with the subspace $J\left(X_{1} \times X_{2}\right) \subset L^{0}\left(\mu_{1} \otimes \mu_{2}\right)$, which is the linear span of the functions $f \times g$. So from from now on we will write $f_{1} \otimes f_{2}$ instead of $f_{1} \times f_{2}$ and consider

$$
X_{1}\left(\mu_{1}\right) \otimes X_{2}\left(\mu_{2}\right)=\left\{\sum_{n=1}^{N} f_{n} \otimes g_{n}: N \in \mathbb{N}, f_{n} \in X_{1}, g_{n} \in X_{2}, n=1, \ldots, N\right\} .
$$




\section{The COMPLETION OF NORMED SPACES OF MEASURABLE FUnCTIONS With THE SUBSEQUENCE PROPERTY}

Since the spaces $X(\mu)$ we will study may not be complete, it is fairly possible that its normed completion may not be described as a vector subspace of $L^{0}(\mu)$. This takes us to consider the condition we have called $L^{0}(\mu)$-completability.

We will start this section by extending Proposition 2.2 to the $\sigma$-finite case. Surely this result is well known but we did not find an adequate reference for it (compare to [1, Section 1.6: Thm. 1.82, Exercise 1.6.11]).

Let $(\Omega, \Sigma, \mu)$ be a $\sigma$-finite measure space with $\mu(\Omega)=\infty$. Express $\Omega=\bigcup_{n=1}^{\infty} \Omega_{n}$, where $0<\mu\left(\Omega_{n}\right)<\infty, \forall n \in \mathbb{N}$ and $\Omega_{m} \cap \Omega_{n}=\emptyset$ if $m \neq n$. For each $n \in \mathbb{N}$, let $\Sigma_{n}=\left\{A \in \Sigma: A \subset \Omega_{n}\right\}, \mu_{n}=\mu: \Sigma_{n} \rightarrow[0, \infty)$ and $d_{n}$ the distance in $L^{0}\left(\Omega_{n}\right)$ given as in (2.1). In what follows when we consider a function $h: \Omega \rightarrow \mathbb{R}$ as having $\Omega_{n}$ as its domain, we will understand that we refer to its restriction to $\Omega_{n}$.

For $f, g \in L^{0}(\mu)$ we now define

$$
d(f, g):=\sum_{n=1}^{\infty} \frac{1}{2^{n}} \frac{d_{n}(f, g)}{\mu\left(\Omega_{n}\right)} .
$$

Since $d_{n}(f, g) \leq \mu\left(\Omega_{n}\right), \forall n \in \mathbb{N}$, the above series always converges. Moreover it defines a distance $d$ in $L^{0}(\mu)$ such that, for $\left\{f_{k}\right\} \subset L^{0}(\mu)$ and $f \in L^{0}(\mu)$, we have

$$
f_{k} \stackrel{L^{0}(\mu)}{\longrightarrow} f \text { if, and only if } f_{k} \stackrel{L^{0}\left(\mu_{n}\right)}{\longrightarrow} f, \forall n \in \mathbb{N} \text {. }
$$

From now on when we consider $L^{0}(\mu)$ as a metric space it will always be with the distance $d$ defined in (3.1). Notice that $d$ is translation invariant and $L^{0}(\mu)$ turns out to be a complete metric space.

Lemma 3.1. The metric space $L^{0}(\mu)$ has the subsequence property.

Proof. Let us take a sequence $\left\{f_{k}\right\} \subset L^{0}(\mu)$ and $f \in L^{0}(\mu)$ such that $f_{k} \stackrel{L^{0}(\mu)}{\longrightarrow} f$. This implies that $\left\{f_{k}\right\}$ converges to $f$ in $L^{0}\left(\mu_{1}\right)$. Since $\mu\left(\Omega_{1}\right)<\infty$, it follows there is some subsequence $\left\{f_{1, \ell}\right\}$ of $\left\{f_{k}\right\}$ such that $\left\{f_{1, \ell}\right\}$ converges pointwise to $f \mu$ a.e. on $\Omega_{1}$. We next apply in $L^{0}\left(\mu_{2}\right)$ the same argument with $\left\{f_{1, \ell}\right\}$ to obtain a subsequence $\left\{f_{2, \ell}\right\}$ of $\left\{f_{1, \ell}\right\}$ such that $\left\{f_{2, \ell}\right\}$ converges pointwise to $f \mu$-a.e. on $\Omega_{2}$. Note that being a subsequence of $\left\{f_{1, \ell}\right\}$, this subsequence $\left\{f_{2, \ell}\right\}$ also converges to $f \mu$-a.e. on $\Omega_{1}$. Repeating this argument, we obtain a family of sequences $\left\{f_{n, \ell}\right\}_{\ell}$ such that $\left\{f_{n+1}, \ell\right\}_{\ell}$ is a subsequence of $\left\{f_{n, \ell}\right\}$ and, for each $n \in \mathbb{N}, f_{n, \ell} \rightarrow f$ pointwise $\mu$-a.e. on $\Omega_{n}$, when $\ell \rightarrow \infty$. We now use a diagonal process and define $g_{n}=f_{n, n}, \forall n \in \mathbb{N}$. Then $\left\{g_{n}\right\}$ is a subsequence of $\left\{f_{k}\right\}$ and $g_{n} \rightarrow f$ pointwise $\mu$-a.e. on each set $\Omega_{n}$. Thus we obtain the conclusion.

Proposition 3.2. A metric space $M=M(\mu)$ has the subsequence property if, and only if, it is continuously included in $L^{0}(\mu)$.

Proof. Let $j: M \rightarrow L^{0}(\mu)$ be the inclusion map. We will first assume that $M$ has the subsequence property. Take $f \in M$ and a sequence $\left\{f_{k}\right\} \subset M$ such that $f_{k} \stackrel{M}{\rightarrow} f$. Fix $k \in \mathbb{N}$ and consider a subsequence $\left\{f_{1, \ell}\right\}$ of $\left\{f_{k}\right\}$. Then also $f_{1, \ell} \stackrel{X}{\rightarrow} f$ and so the subsequence property implies there is some subsequence $\left\{f_{2, \ell}\right\}$ of $\left\{f_{1, \ell}\right\}$ 
which converges pointwise to $f \mu$-a.e. on $\Omega_{n}$. Applying Lemma (2.1) it follows that $\left\{f_{k}\right\}$ converges to $f$ in $L^{0}\left(\mu_{n}\right)$. Using (3.2), we now conclude $f_{k} \stackrel{L^{0}(\mu)}{\longrightarrow} f$.

Let us now assume that $j$ is continuous and take $\left\{f_{k}\right\} \subset M$ and $f \in M$ such that $f_{k} \stackrel{M}{\rightarrow} f$. The continuity of $j$ implies now that $f_{k} \stackrel{L^{0}(\mu)}{\longrightarrow} f$. Applying now Lemma 3.1 we obtain the conclusion.

We next show that the convergence in $L^{0}(\mu)$ does not depend on the partition of $\Omega$ that was initially employed to define in (3.1) the distance $d$. So let $\left\{A_{k}\right\} \subset \Sigma$ be a collection such that $\bigcup_{k=1}^{\infty} A_{k}=\Omega, A_{k} \cap A_{m}=\emptyset$ if $k \neq m$, and $\mu\left(A_{k}\right)<\infty$, $\forall k \in \mathbb{N}$. For each $k \in \mathbb{N}$, let us consider the measure space $\left(A_{k}, \Sigma_{k}, \mu\right)$ where $\Sigma_{k}=\left\{A \in \Sigma: A \subset \Omega_{k}\right\}$ and take as $\rho_{k}$ the corresponding distance defined by (2.1). Let $\rho$ denote the distance in $L^{0}(\mu)$ that we obtain in this way. As before, when we treat a function $h: \Omega \rightarrow \mathbb{R}$ as having $A_{k}$ as its domain, we will understand that we refer to its restriction to $A_{k}$.

Corollary 3.3. Let $M=M(\mu)$ be a metric space, $\left\{f_{n}\right\} \subset M$ and $f \in M$. Then $\rho_{k}\left(f_{n}, f\right) \rightarrow 0, \forall k \in \mathbb{N}$ if, and only if $f_{n} \stackrel{L^{0}(\mu)}{\longrightarrow} f$.

Proof. We will start showing that $\left(L^{0}(\mu), \rho\right)$ has the subsequence property. So take $\left\{g_{n}\right\} \subset L^{0}(\mu)$ and $g \in L^{0}(\mu)$ such that $g_{n} \stackrel{\rho}{\rightarrow} g$. Using now Proposition 3.2 (with respect to the distance $\rho$ ) we conclude that there is a subsequence $\left\{g_{n(k)}\right\}$ that converges pointwise to $g \mu$-a.e.. Applying again Proposition 3.2 this implies the inclusion $j_{\rho}:\left(L^{0}(\mu), \rho\right) \rightarrow L^{0}(\mu, d)$ is continuous.

Since the above argument is symmetric with respect to $d$ and $\rho$, it follows that also the inclusion $j_{d}: L^{0}(\mu, d) \rightarrow L^{0}(\mu, \rho)$ is continuous.

Finally, we just have to notice that $\rho_{k}\left(f_{n}, f\right) \rightarrow 0, \forall k \in \mathbb{N}$ if, and only if, $\rho\left(f_{n}, f\right) \rightarrow f$.

Now, we introduce one of the main definitions of the paper: the $L^{0}$-completability of a normed space. Essentially, it means that the closure of this normed space is included in $L^{0}(\mu)$.

Definition 3.4. A normed space $X=X(\mu)$ is $L^{0}(\mu)$-completable (or simply $L^{0}$ completable) when it has the following properties:

a) $X$ has the subsequence property.

b) If $\left\{f_{n}\right\}$ is a Cauchy sequence in $X$ and $\left\{f_{n}\right\}$ converges to 0 pointwise $\mu$-a.e., then $\left\|f_{n}\right\|_{X} \rightarrow 0$.

Definition 3.5. Let $X=X(\mu)$ be an $L^{0}(\mu)$-completable normed space and take $\widehat{X}=\widehat{X}(\mu)$ to consist of all functions $f \in L^{0}(\mu)$ for which there is a Cauchy sequence $\left\{f_{n}\right\} \subset X$ such that $f_{n} \rightarrow f$ pointwise $\mu$-a.e.. For a given $f \in \widehat{X}$ the expression

$$
\|f\|_{\widehat{X}}:=\lim _{n \rightarrow \infty}\left\|f_{n}\right\|_{X} .
$$

defines a norm on $\widehat{X}$.

Indeed, assume first that $\left\{f_{n}\right\}$ and $\left\{g_{n}\right\}$ are Cauchy sequences in $X$ such that both $f_{n} \rightarrow f$ and $g_{n} \rightarrow f$ pointwise $\mu$-a.e.. Then $\left\{g_{n}-f_{n}\right\}$ is also a Cauchy 
sequence in $X$ and $g_{n}-f_{n} \rightarrow 0 \mu$-a.e.. Using now b) in Definition 3.4, we have that $\left\|f_{n}-g_{n}\right\|_{X} \rightarrow 0$. This implies that the function $\|\cdot\|_{\widehat{X}}$ is well defined and by proceeding directly we can check it is a seminorm on the vector space $\widehat{X}$. To prove that it is a norm, let us suppose that $f \in \widehat{X}$ and $\|f\|_{\widehat{X}}=0$. Hence there is a Cauchy sequence $\left\{f_{n}\right\} \subset X$ such that $f_{n} \rightarrow f$ pointwise $\mu$-a.e. and $\left\|f_{n}\right\|_{X} \rightarrow 0$. By property a) in Definition 3.4 it follows that $f_{n} \stackrel{L^{0}(\mu)}{\longrightarrow} 0$. Hence $f_{n(k)} \rightarrow 0$ pointwise $\mu$-a.e., for some subsequence $\left\{f_{n(k)}\right\}$ of $\left\{f_{n}\right\}$. This allows us to conclude that $f=0 \mu$-a.e..

Notice that

$$
X \subset \widehat{X} \text { and }\|f\|_{\widehat{X}}=\|f\|_{X}, \forall f \in X .
$$

As we said before, in our next result we prove that if a normed space $X$, is $L^{0}$-completable then its completion is actually $\widehat{X}$ and, in addition, it has the subsequence property.

Theorem 3.6. Let $X(\mu)$ be an $L^{0}(\mu)$-completable normed space. Then:

i) Its completion is $\widehat{X}=\widehat{X}(\mu)$.

ii) $\widehat{X}$ has the subsequence property.

Proof. i) Take $f \in \widehat{X}$ and consider a Cauchy sequence $\left\{f_{n}\right\} \subset X$ such that $f_{n} \rightarrow f$ pointwise $\mu$-a.e.. We will show that

$$
f_{n} \stackrel{\widehat{X}}{\rightarrow} f .
$$

Consider $\epsilon>0$ and take $N \in \mathbb{N}$ to be such that $\left\|f_{n}-f_{m}\right\|_{X} \leq \epsilon, \forall m, n \geq N$. Fix $n \geq N$. Then $\left\{f_{n}-f_{m}\right\}_{m}$ is a Cauchy sequence in $X$ that converges to $f_{n}-f$ pointwise $\mu$-a.e.. Hence $\left\|f_{n}-f\right\|_{\widehat{X}}=\lim _{m \rightarrow \infty}\left\|f_{n}-f_{m}\right\|_{X} \leq \epsilon, \forall n \geq N$. This establishes (3.4). Note that this indicates that $X$ is dense in $\widehat{X}$.

To establish that $\widehat{X}$ is a Banach space, let $\left\{g_{n}\right\}$ be a Cauchy sequence in $\widehat{X}$. Then, for each $n \in \mathbb{N}$ we can find a function $f_{n} \in X$ such that $\left\|f_{n}-g_{n}\right\|_{\widehat{X}} \leq \frac{1}{n}$. It follows that $\left\{f_{n}\right\}$ is a Cauchy sequence in $X$. So there is some subsequence $\left\{f_{n(k)}\right\}$ that converges pointwise $\mu$-a.e., say to $g$. Then $g \in \widehat{X}$ and the conclusion will follow by showing that $g_{n(k)} \stackrel{\widehat{X}}{\rightarrow} g$. Using (3.4) we obtain that

$$
\left\|g-g_{n(k)}\right\|_{\widehat{X}} \leq\left\|g-f_{n(k)}\right\|_{\widehat{X}}+\frac{1}{n(k)} \rightarrow 0, \quad \text { when } k \rightarrow \infty .
$$

ii) By Proposition 3.2 we will show that the inclusion $j: \widehat{X} \rightarrow L^{0}(\mu)$ is continuous. Let $\left\{g_{k}\right\} \subset \widehat{X}$ and $g \in \widehat{X}$ satisfy $g_{k} \stackrel{\widehat{X}}{\rightarrow} g$. Applying (3.2), to conclude that $g_{k} \stackrel{L^{0}(\mu)}{\longrightarrow} g$ we will establish that $g_{k} \stackrel{L^{0}\left(\mu_{n}\right)}{\longrightarrow} g$, for each $n \in \mathbb{N}$.

For each $k \in \mathbb{N}$ take a sequence $\left\{f_{k, \ell}\right\}_{\ell} \subset X$ such that when $\ell \rightarrow \infty$ we have

$$
f_{k, \ell} \stackrel{\widehat{X}}{\rightarrow} g_{k} \text { and } f_{k, \ell} \rightarrow g_{k} \text { pointwise } \mu-\text { a.e.. }
$$

By Proposition 3.2, the normed space $X$ is continuously included in $L^{0}(\mu)$. So, given $\epsilon>0$, there is some $\delta>0$ such that

$$
\text { if } f \in X \text { and }\|f\|_{X} \leq \delta \text {, then } d(f, 0)<\frac{\epsilon}{2} \text {. }
$$


Let us now fix $n \in \mathbb{N}$ and consider $k \in \mathbb{N}$. Since $\mu\left(\Omega_{n}\right)<\infty$ and $f_{k, \ell} \rightarrow g_{k}$ pointwise $\mu$-a.e. when $\ell \rightarrow \infty$, it follows from (2.1) that $d_{n}\left(g_{k}, f_{k, \ell}\right)=0$.

Choose $\ell(k)$ so that for $h_{k}=f_{k, \ell(k)} \in X$ we have $\left\|h_{k}\right\|_{X} \leq\left\|f_{k}\right\|_{\widehat{X}}+\frac{1}{k}$ and $d\left(g_{k}, h_{k}\right)<\frac{\epsilon}{2}$. Take $K \in \mathbb{N}$ such that $\left\|h_{k}\right\|_{X} \leq \delta, \forall k \geq K$. Then, for $k \geq K$ from (3.6) we have $d_{n}\left(g_{k}, h_{k}\right) \leq \frac{\epsilon}{2}$. Therefore

$$
d_{n}\left(g_{k}, f\right) \leq d_{n}\left(g_{k}, h_{k}\right)+d\left(h_{k}, f\right) \leq \frac{\epsilon}{2}+\frac{\epsilon}{2}=\epsilon, \forall k \geq K .
$$

This proves that $\left\{g_{k}\right\}$ converges to $f$ in $L^{0}\left(\mu_{n}\right)$.

Corollary 3.7. Let $X$ be a subspace of a normed space $Y=Y(\mu)$ having the subsequence property. If $X$ is dense in $Y$ and $X$ is $L^{0}(\mu)$-completable, then also $Y$ is $L^{0}(\mu)$-completable and $\widehat{Y}=\widehat{X}$.

Proof. Let $\left\{f_{n}\right\} \subset Y$ be a Cauchy sequence that converges pointwise to 0 . By the density of $X$ in $Y$, take a sequence $\left\{g_{n}\right\} \subset X$ such that $\left\|g_{n}-f_{n}\right\|_{Y} \leq \frac{1}{n}, \forall n \in \mathbb{N}$. Then $\left\{g_{n}\right\}$ is a Cauchy sequence in $X$. Since $X$ is $L^{0}(\mu)$-completable, this implies that $g_{n} \stackrel{X}{\rightarrow} g$ pointwise $\mu$-a.e., for some $g \in L^{0}(\mu)$. On the other hand, we have $f_{n}-g_{n} \stackrel{Y}{\rightarrow} 0$. So it follows there is some subsequence $\left\{f_{n(k)}-g_{n(k)}\right\}$ that converges to 0 pointwise $\mu$-a.e. Hence $g_{n(k)} \rightarrow 0$ pointwise $\mu$-a.e. and we obtain that $g=0 \mu$ a.e.. Since $X$ is $L^{0}(\mu)$-completable, we conclude that $g_{n} \stackrel{X}{\rightarrow} 0$. Thus we have $f_{n}=\left(f_{n}-g_{n}\right)+g_{n} \stackrel{Y}{\rightarrow} 0$. This shows $X$ is $L^{0}(\mu)$-completable.

Clearly $\widehat{X} \subset \widehat{Y}$. Take $f \in \widehat{Y}$ and let $\left\{f_{n}\right\} \subset Y$ be a Cauchy sequence converging pointwise to $f$. By the density of $X$ in $Y$ choose now a sequence $\left\{g_{n}\right\} \subset X$ such that $\left\|g_{n}-f_{n}\right\|_{Y} \leq \frac{1}{n}, \forall n \in \mathbb{N}$. Then $\left\{g_{n}\right\} \subset X$ is also a Cauchy sequence and there is some subsequence $\left\{g_{n(k)}-f_{n(k)}\right\}$ such that $g_{n(k)}-f_{n(k)} \rightarrow 0 \mu$-a.e.. Hence $g_{n(k)}=f_{n(k)}+\left(g_{n(k)}-f_{n(k)}\right) \rightarrow f$ pointwise $\mu$-a.e. Therefore $f \in \widehat{X}$.

In certain cases, instead of working explicitly in the above context, to describe the completion we can do it in the following more "simple" situation. Recall that $X=X(\Omega)$ just means that $X$ is a normed space consisting of functions $f: \Omega \rightarrow \mathbb{R}$.

Definition 3.8. A normed space $X=X(\Omega)$ is F-completable if:

a) For any $w \in \Omega$ the evaluation map $\delta_{w}: X \rightarrow \mathbb{R}$, given by $f \mapsto f(w)$, is continuous.

b) If $\left\{f_{n}\right\} \subset X$ is a Cauchy sequence that converges pointwise to 0 , then $\left\|f_{n}\right\|_{X} \rightarrow 0$.

As usual, to introduce a normed space $X=X(\Omega)$ into a measure-theoretic context, we consider the measure space $(\Omega, \Sigma, \mu)$, where $\Sigma=2^{\Omega}$ and $\mu$ is the counting measure. When $\Omega$ is a countable set, then we have a $\sigma$-finite measure and $X$ is a normed space of $\mu$-measurable functions.

Theorem 3.9. Let $\Omega$ be a countable set. If a normed space $X=X(\Omega)$ is $F$ completable, then its completion $\widehat{X}$ consists of all functions $f: \Omega \rightarrow \mathbb{R}$ having a Cauchy sequence $\left\{f_{n}\right\} \subset X$ that converges pointwise to $f$. Moreover, in $\widehat{X}$ each evaluation map is continuous.

Proof. Note first that since $X$ is F-completable, then $X$ is $L^{0}(\mu)$-completable. So its completion $\widehat{X}$ is well defined. Let $X_{1}$ be the subspace of $F(\Omega)$ formed by all 
functions $f: \Omega \rightarrow \mathbb{R}$ with a Cauchy sequence $\left\{f_{n}\right\} \subset X$ that converges pointwise to $f$. Clearly $X_{1} \subset \widehat{X}$. Assume now that $f \in \widehat{X}$. Then there is a Cauchy sequence $\left\{f_{n}\right\} \subset X$ having a subsequence $\left\{f_{n(k)}\right\}$ such that $f_{n(k)} \rightarrow f$ pointwise. By property a) in Definition 3.8, it follows that $f_{n(k)} \rightarrow g$ pointwise, for some function $g \in X_{1}$. Hence $f=g \in X_{1}$.

We now prove that each evaluation map is continuous on $\widehat{X}$. Fix $w \in \Omega$. Since $X$ is F-completable, take a real number $C>0$ such that $|f(w)| \leq C\|f\|_{X}, \forall f \in X$. Consider now $g \in \widehat{X}$ and choose a sequence $\left\{f_{n}\right\} \subset X$ such that $f_{n} \stackrel{\widehat{X}}{\rightarrow} g$ and $f_{n}(w) \rightarrow f(w)$. Then $|g(w)|=\lim _{n \rightarrow \infty}\left|f_{n}(w)\right| \leq C \lim _{n \rightarrow \infty}\left\|f_{n}\right\|_{X}=C\|g\|_{\widehat{X}}$.

Corollary 3.10. Let $X$ be a subspace of a normed space $Y=Y(\Omega)$. If each evaluation map is continuous on $Y, X$ is dense in $Y$ and $X$ is F-completable, then also $Y$ is $F$-completable and $\widehat{Y}=\widehat{X}$.

Proof. Let $\left\{f_{n}\right\} \subset Y$ be a Cauchy sequence that converges pointwise to 0 . By the density of $X$ in $Y$, take a sequence $\left\{g_{n}\right\} \subset X$ such that $\left\|g_{n}-f_{n}\right\|_{Y} \leq \frac{1}{n}, \forall n \in \mathbb{N}$. Then $\left\{g_{n}\right\}$ is a Cauchy sequence in $X$. Let $w \in \Omega$. Since

$$
\left|g_{n}(w)\right| \leq\left|g_{n}(w)-f_{n}(w)\right|+\left|f_{n}(w)\right| \leq \frac{C_{w}}{n}+\left|f_{n}(w)\right|,
$$

it follows that $g_{n} \rightarrow 0$ pointwise. Since $X$ is $F$-completable, this implies that $\left\|g_{n}\right\|_{Y}=\left\|g_{n}\right\|_{X} \rightarrow 0$. Noting now that $\left\|f_{n}\right\|_{Y} \leq\left\|f_{n}-g_{n}\right\|_{Y}+\left\|g_{n}\right\|_{Y} \leq \frac{1}{n}+\left\|g_{n}\right\|_{Y}$, we conclude that $\left\|f_{n}\right\|_{Y} \rightarrow 0$. This shows $Y$ is F-completable.

By applying Corollary 3.7 we obtain $\widehat{Y}=\widehat{X}$.

The next result gives examples of normed sequence spaces that do not have the subsequence property with respect to the counting measure.

Example 3.11. Let $X$ be any normed sequence space containing the canonical system $\left\{e_{n}: n \in \mathbb{N}\right\}$ as a bounded set. For each $N \in \mathbb{N}$, let $v_{N}=\sum_{n=1}^{N} e_{n}$. Clearly, the set $\left\{v_{N}: N \in \mathbb{N}\right\}$ is linearly independent. We now define

$$
T\left(v_{N}\right)=\frac{1}{N} e_{N}, \forall N \in \mathbb{N},
$$

and extend it linearly to the linear span $Y$ of $\left\{v_{N}: N \in \mathbb{N}\right\}$. In this way we obtain an injective linear operator $T: Y \rightarrow X$. Hence the function

$$
\|y\|=\|T y\|_{X},
$$

is a norm on $Y$. From (3.7) and (3.8) it follows that $v_{N} \stackrel{Y}{\rightarrow} 0$.

On the other side, let $\left\{v_{N(k)}\right\}$ be any subsequence of $\left\{v_{N}\right\}$. Then $V_{N(k)}(j) \rightarrow 1$, $\forall j \in \mathbb{N}$.

\section{2-RECtangular NORMED SPACES OF MEASURABle FUnCtions}

Let $\left(\Omega_{1}, \Sigma_{1}, \mu_{1}\right)$ and $\left(\Omega_{2}, \Sigma_{2}, \mu_{2}\right)$ be $\sigma$-finite measure spaces. In the following we will work in $\left(\Omega_{1} \times \Omega_{2}, \Sigma_{1} \otimes \Sigma_{2}, \mu_{1} \otimes \mu_{2}\right)$, which also is a $\sigma$-finite measure space. Although we are mainly interested in working with the specific semiring $\Sigma_{1} \widetilde{\times} \Sigma_{2}$, it will be convenient to make our definition with respect to an arbitrary semiring 
$\mathcal{S} \subset \Sigma_{1} \otimes \Sigma_{2}$. Have in mind that, to abbreviate, we frequently take $\Omega=\Omega_{1} \times \Omega_{2}$, $\Sigma=\Sigma_{1} \otimes \Sigma_{2}$ and $\mu=\mu_{1} \otimes \mu_{2}$.

Definition 4.1. A normed space $Z=Z\left(\mu_{1} \otimes \mu_{2}\right)$ is a 2-rectangular normed space (2-rectangular n.s.) if for $f \in Z, R \in \mathcal{S}$ we always have $\chi_{R} f \in Z$ and there is some real number $C>0$ such that

$$
\left\|\chi_{R} f\right\|_{Z} \leq C\|f\|_{Z}
$$

Usually we will not mention the semiring $\mathcal{S}$. So the norm will just be called 2-rectangular and we will say that $C$ is a 2-rectangularity constant for $Z$.

Lemma 4.2. If $X=X\left(\mu_{1} \otimes \mu_{2}\right)$ is $L^{0}(\mu)$-completable and 2-rectangular, then its completion $\hat{X}$ is also 2-rectangular.

Proof. Take $C>0$ as a rectangularity constant for $X$. Let $R \in \mathcal{S}$ and $f \in \widehat{X}$. Hence there is a Cauchy sequence $\left\{f_{n}\right\} \subset X$ such that $f_{n} \rightarrow f$ pointwise $\mu$-a.e. Since $X$ is 2-rectangular, it follows that $\left\{\chi_{R} f_{n}\right\} \subset X$ is also a Cauchy sequence. Moreover, $\chi_{R} f_{n} \rightarrow \chi_{R} f$ pointwise $\mu$-a.e.. Thus $\chi_{R} f=g \in \widehat{X}$ and

$$
\left\|\chi_{R} f\right\|_{\widehat{X}}=\lim _{n \rightarrow \infty}\left\|\chi_{R} f_{n}\right\|_{X} \leq C \lim _{n \rightarrow \infty}\left\|f_{n}\right\|_{X}=C\|f\|_{\widehat{X}} .
$$

Given $f \in F\left(\Omega_{1}\right)$ and $g \in F\left(\Omega_{2}\right)$, recall that $f \otimes g: \Omega \rightarrow \mathbb{R}$ is given by

$$
f \otimes g(x, y):=f(x) g(y) \text {. }
$$

Then, for $A \in \Sigma_{1}$ and $B \in \Sigma_{2}$, we have $\chi_{A} \otimes \chi_{B}=\chi_{A \times B}$.

Lemma 4.3. If $X_{1}=X_{1}\left(\mu_{1}\right)$ and $X_{2}=X_{2}\left(\mu_{2}\right)$ are 1-rectangular n.s. with respect to correspondent semirings $\mathcal{S}_{1}$ and $\mathcal{S}_{2}$, and $q$ is a uniform reasonable crossnorm on $X_{1} \otimes X_{2}$, then $X_{1} \otimes_{q} X_{2}$ is 2-rectangular with respect to the semiring $\mathcal{S}_{1} \widetilde{\times} \mathcal{S}_{2}$. Moreover, if $C_{1}$ is a constant of 1-rectangularity for $X_{1}$ and $C_{2}$ is a constant of 1-rectangularity for $X_{2}$, then $C_{1} C_{2}$ is a constant of 2-rectangularity for $X_{1} \otimes X_{2}$.

Proof. Let $R=A \times B$, where $A \in \mathcal{S}_{1}$ and $B \in \mathcal{S}_{2}$. Take $t \in X_{1} \otimes X_{2}$ and note that $\chi_{R} t=\left(\chi_{A} \otimes \chi_{B}\right) t=\left(M_{A} \otimes M_{B}\right)(t)$, where $M_{A} f=\chi_{A} f, \forall f \in X$ and $M_{B} g=\chi_{B} g, \forall g \in Y$. By the hypothesis we have that $\left\|M_{A} f\right\| \leq C_{1}\|f\|, \forall f \in X$ and $\left\|M_{B} g\right\| \leq C_{2}\|g\|, \forall g \in Y$. Since $q$ is a uniform norm, this implies that $\left\|M_{A} \otimes M_{B}\right\| \leq C_{1} C_{2}$ and the conclusion follows.

Since a Banach function space $X(\mu)$ is always a 1-rectangular normed space with 1 as a constant of rectangularity, from Lemma 4.3 follows directly the following.

Lemma 4.4. If $X_{1}=X\left(\mu_{1}\right)$ and $X_{2}=X\left(\mu_{2}\right)$ are Banach function spaces and $q$ is a uniform reasonable cross norm on $X_{1} \otimes X_{2}$, then $X\left(\mu_{1}\right) \otimes_{q} X\left(\mu_{2}\right)$ is a 2rectangular n.s., with 1 as a constant of regularity.

In what follows we show some easy results regarding inclusions of 2-rectangular tensor product (without completion) over the product measure. These results will be used in Section 5 . 
Proposition 4.5. If $X_{1}=X_{1}\left(\mu_{1}\right)$ and $X_{2}=X_{2}\left(\mu_{2}\right)$ are B.f.s. consisting of locally integrable functions, then $X_{1} \otimes_{\pi} X_{2}$ is continuously included into $L^{0}\left(\mu_{1} \otimes \mu_{2}\right)$ and has the $\mu_{1} \otimes \mu_{2}$-subsequence property.

Proof. Let $\left\{t_{n}\right\} \subset X_{1} \otimes_{\pi} X_{2}$ and $t \in X_{1} \otimes_{\pi} X_{2}$ satisfy $t_{n} \stackrel{\pi}{\rightarrow} t$. We now fix $A \in \Sigma_{1}$, $B \in \Sigma_{2}$ such that $\mu_{1}(A)<\infty, \mu_{2}(B)<\infty$. Next, for $f \in X_{1}, g \in X_{2}$ define $T f=\chi_{A} f$ and $S g=\chi_{B} g$. By our hypothesis we have $T: X_{1} \rightarrow L^{1}\left(\mu_{1}\right)$ and $S: X_{2} \rightarrow L^{1}\left(\mu_{2}\right)$. Since $T$ and $S$ are positive linear operators between Banach lattices, it follows that each of them is bounded. Let $C_{j}=\left\|T_{j}\right\|, j=1,2$.

Take $R=A \times B$. Given $f_{j} \in X_{1}, g \in X_{2}$, let $B(f, g)=\chi_{A} f \otimes \chi_{B} g$. Then $B: X_{1} \times X_{2} \rightarrow L^{1}\left(\mu_{1} \otimes \mu_{2}\right)$ is a bilinear map. Applying Fubini's Theorem we find $\left\|\chi_{R} f \otimes g\right\|_{L^{1}(\mu)} \leq\left\|\chi_{A} f\right\|_{L^{1}\left(\mu_{1}\right)}\left\|\chi_{B} g\right\|_{L^{1}\left(\mu_{2}\right)} \leq C_{1} C_{2}\|f\|_{X_{1}}\|g\|_{X_{2}}, \forall f \in X_{1}, g \in X_{2}$.

By the fundamental property of the projective norm, this implies $\chi_{R} t \in L^{1}(\mu)$, $\forall t \in X \otimes Y$ and

$$
\left\|\chi_{R} t\right\|_{L^{1}\left(\mu_{1} \otimes \mu_{2}\right)} \leq C_{1} C_{2}\|t\|_{\pi}, \forall t \in X_{1} \otimes X_{2} .
$$

Let $j=1,2$. Since $\mu_{j}$ is a $\sigma$-finite measure, we express $\Omega_{j}=\cup_{m=1}^{\infty} \Omega_{j, m}$, where $\mu_{j}\left(\Omega_{j, m}\right)<\infty, \forall m \in \mathbb{N}$. Notice that $\mathcal{S}_{j}=\left\{A \in \Sigma_{j}: \mu_{j}(A)<\infty\right\}$ is a ring of sets in $\Omega_{j}$. Then $\Omega=\Omega_{1} \times \Omega_{2}=\bigcup_{m, n \in \mathbb{N}} \Omega_{1, m} \times \Omega_{2, n}$. Since $\mathcal{S}_{1} \widetilde{\times} \mathcal{S}_{2}$ is a semiring, it follows that we can represent $\Omega=\bigcup_{k=1}^{\infty} R_{k}$, where $R_{k} \in \mathcal{S}_{1} \widetilde{\times} \mathcal{S}_{2}, \forall k \in \mathbb{N}$ and $R_{k} \cap R_{\ell}=\emptyset$ when $k \neq \ell$.

From (4.2) it follows that for each $R=A \times B$ with $\mu_{1} \otimes \mu_{2}(R)<\infty$ there is a continuous projection on $X_{1} \otimes_{\pi} X_{2}$ whose image is contained in $L^{0}\left(\mu_{R}\right)$. Applying now Corollary 3.3 we obtain that $X_{1} \otimes_{\pi} X_{2}$ is continuously included into $L^{0}\left(\mu_{1} \otimes \mu_{2}\right)$. Hence, by Proposition 3.2, $X_{1} \otimes_{\pi} X_{2}$ has the subsequence property.

In relation to the following result see [17, Thm. 4.5.1].

Proposition 4.6. Let $1 \leq p, s \leq \infty$ satisfy $\frac{1}{r}=\frac{1}{p}+\frac{1}{s} \leq 1$ and $X_{1}=X_{1}\left(\mu_{1}\right)$, $X_{2}=X_{2}\left(\mu_{2}\right)$ be Banach function spaces. If $\mu_{1}$ and $\mu_{2}$ are finite measures, $X_{1} \subset L^{p}\left(\mu_{1}\right)$ and $X_{2} \subset L^{s}\left(\mu_{2}\right)$, then $X_{1} \otimes_{\pi} X_{2} \subset L^{r}\left(\mu_{1} \otimes \mu_{2}\right)$ and the inclusion is continuous.

Proof. Given $f: \Omega_{1} \rightarrow \mathbb{R}$, consider $\tilde{f}=f \otimes 1: \Omega \rightarrow \mathbb{R}$. Similarly, for $g: \Omega_{2} \rightarrow \mathbb{R}$ we have $\widetilde{g}=1 \otimes g: \Omega \rightarrow \mathbb{R}$. Then we have that $\widetilde{f} \in L^{p}\left(\mu_{1} \otimes \mu_{2}\right)$. Similarly, we have that $\widetilde{g} \in L^{s}\left(\mu_{1} \otimes \mu_{2}\right)$. Hence, using Hölder's inequality, we have $f \otimes g=\widetilde{f} \widetilde{g} \in L^{r}\left(\mu_{1} \otimes \mu_{2}\right)$.

Consider now the map $B: X \times Y \rightarrow L^{r}\left(\mu_{1} \otimes \mu_{2}\right)$ defined by $B(f, g)=f \otimes g$. Since $B$ is positive bilinear map, it is continuous, as in the linear case; the proof of this fact can be easily derived from the argument for the linear case, that can be found in $[15$, p.2]. By the fundamental property of the projective norm this implies that $\|t\|_{L^{r}\left(\mu_{1} \otimes \mu_{2}\right)} \leq\|B\|\|t\|_{\pi}, \forall t \in X \otimes_{\pi} Y$.

A direct argument allows to prove the next result.

Proposition 4.7. Let $p \geq 1$ and $X_{1}=X_{1}\left(\mu_{1}\right), X_{2}=X_{2}\left(\mu_{2}\right)$ be Banach function spaces. If $X_{1} \subset L^{p}\left(\mu_{1}\right)$ and $X_{2} \subset L^{p}\left(\mu_{2}\right)$, then $X_{1} \otimes_{\pi} X_{2} \subset L^{p}\left(\mu_{1} \otimes \mu_{2}\right)$ and the inclusion is continuous. 
Following the proof of Proposition 4.6 we obtain our next result.

Lemma 4.8. Let $X_{1}=X_{1}\left(\mu_{1}\right), X_{2}=X_{2}\left(\mu_{2}\right)$ and $X_{3}=X_{3}\left(\mu_{1} \otimes \mu_{2}\right)$ be Banach function spaces. If $X_{1} \otimes X_{2} \subset X_{3}$, then the inclusion $j: X_{1} \otimes_{\pi} X_{2} \rightarrow X_{3}$ is continuous.

Proof. Let $B: X_{1} \times X_{2} \rightarrow X_{3}$ be defined as $B(f, g)=f \otimes g$. Since $B$ is a positive bilinear map, then $B$ is continuous. Applying now the fundamental property of the projective norm we obtain the conclusion.

\section{Completability of the projective tensor product of Banach FUNCTION SPACES WITH A SCHAUdER BASIS}

Let $\left(\Omega_{1}, \Sigma_{1}, \mu_{1}\right)$ and $\left(\Omega_{2}, \Sigma_{2}, \mu_{2}\right)$ be $\sigma$-finite measure spaces. We will assume that both $X_{1}=X_{1}\left(\mu_{1}\right)$ and $X_{2}=X_{2}\left(\mu_{2}\right)$ are B.f.s. with a Schauder basis. So, let $\left\{f_{j}: j \in \mathbb{N}\right\}$ be a Schauder basis for $X_{1}$ and $\left\{f_{j}^{*}\right\}$ be its biorthogonal basic sequence. Similarly, let $\left\{g_{k}: k \in \mathbb{N}\right\}$ be a Schauder basis for $X_{2}$ and $\left\{g_{k}^{*}: k \in \mathbb{N}\right\}$ be its biorthogonal basic sequence.

Given $n \in \mathbb{N}$, let $P_{n}: X_{1}\left(\mu_{1}\right) \rightarrow X_{1}\left(\mu_{1}\right)$ and $Q_{n}: X_{2}\left(\mu_{2}\right) \rightarrow X_{2}\left(\mu_{2}\right)$ be the respective $n$ th- canonical projection. That is,

$$
P_{n}(f)=\sum_{j=1}^{n} f_{j}^{*}(f) f_{j}, \forall f \in X_{1}\left(\mu_{1}\right) ; \quad Q_{n}(g)=\sum_{k=1}^{n} g_{k}^{*}(g) g_{k}, \forall g \in X_{2}\left(\mu_{2}\right) .
$$

Next we consider $R_{n}=P_{n} \otimes Q_{n}: X_{1}\left(\mu_{1}\right) \otimes_{\pi} X_{2}\left(\mu_{2}\right) \rightarrow X_{1}\left(\mu_{1}\right) \otimes_{\pi} X_{2}\left(\mu_{2}\right)$.

It is well known that $\left\{P_{n}\right\}$ and $\left\{Q_{n}\right\}$ are bounded sequences. Hence we fix $C>0$ such that

$$
\left\|P_{n}\right\| \leq C, \quad\left\|Q_{n}\right\| \leq C, \forall n \in \mathbb{N}
$$

Since the projective norm is always a uniform norm, this implies that

$$
\left\|R_{n}\right\| \leq C^{2}, \forall n \in \mathbb{N}
$$

Now we have $P_{n} f \rightarrow f, \forall f \in X_{1}$ and $Q_{n} g \rightarrow g, \forall g \in X_{2}$. This gives that $R_{n}(f \otimes g)=P_{n} f \otimes Q_{n} g \rightarrow f \otimes g, \forall f \in X_{1}, g \in X_{2}$. Thus

$$
R_{n}(t) \rightarrow t, \forall t \in X_{1}\left(\mu_{1}\right) \otimes X_{2}\left(\mu_{2}\right) .
$$

Next, we will establish

$$
R_{n}(t)=\sum_{1 \leq j, k \leq n} f_{j}^{*} \otimes g_{k}^{*}(t) f_{j} \otimes g_{k}, \forall t \in X_{1}\left(\mu_{1}\right) \otimes X_{2}\left(\mu_{2}\right)
$$


Proof. Let $t=\sum_{\ell=1}^{L} f_{\ell} \otimes g_{\ell}$, where $f_{\ell} \in X_{1}, g_{\ell} \in X_{2}, \ell=1, \ldots, L$. Then

$$
\begin{aligned}
R_{n}(t) & =\sum_{\ell=1}^{L} P_{n}\left(f_{\ell}\right) \otimes Q_{n}\left(g_{\ell}\right)=\sum_{\ell=1}^{L}\left[\left(\sum_{j=1}^{n} f_{j}^{*}\left(f_{\ell}\right) f_{j}\right) \otimes\left(\sum_{k=1}^{n} g_{k}^{*}\left(g_{\ell}\right) g_{k}\right)\right] \\
& =\sum_{\ell=1}^{L}\left[\sum_{1 \leq j, k \leq n} f_{j}^{*}\left(f_{\ell}\right) g_{k}^{*}\left(g_{\ell}\right) f_{j} \otimes g_{k}\right]=\sum_{1 \leq j, k \leq n}\left(\sum_{\ell=1}^{L} f_{j}^{*}\left(f_{\ell}\right) g_{k}^{*}\left(g_{\ell}\right)\right) f_{j} \otimes g_{k} \\
& =\sum_{1 \leq j, k \leq n}\left(f_{j}^{*} \otimes g_{k}^{*}\left(\sum_{\ell=1}^{L} f_{\ell} \otimes g_{\ell}\right)\right) f_{j} \otimes g_{k}=\sum_{1 \leq j, k \leq n} f_{j}^{*} \otimes g_{k}^{*}(t) f_{j} \otimes g_{k} .
\end{aligned}
$$

Lemma 5.1. If for any bounded sequence $\left\{t_{m}\right\} \subset X_{1}\left(\mu_{1}\right) \otimes_{\pi} X_{2}\left(\mu_{2}\right)$ such that $t_{m} \rightarrow 0 \mu_{1} \otimes \mu_{2}$-a.e., we have $f_{j}^{*} \otimes g_{k}^{*}\left(t_{m}\right) \rightarrow 0, \forall j, k \in \mathbb{N}$, then for any Cauchy sequence $\left\{t_{m}\right\} \subset X_{1}\left(\mu_{1}\right) \otimes_{\pi} X_{2}\left(\mu_{2}\right)$ such that $t_{m} \rightarrow 0 \mu_{1} \otimes \mu_{2}$-a.e., we have $t_{m} \stackrel{\pi}{\rightarrow} 0$.

Proof. Let $\left\{t_{m}\right\} \subset X_{1} \otimes_{\pi} X_{2}$ be a Cauchy sequence such that $t_{m} \rightarrow 0, \mu_{1} \otimes \mu_{2}$-a.e.. Then $\left\{t_{m}\right\}$ is bounded and by the hypothesis we have

$$
f_{j}^{*} \otimes g_{k}^{*}\left(t_{m}\right) \rightarrow 0, \forall j, k \in \mathbb{N} .
$$

We will now show that $t_{m} \stackrel{\pi}{\rightarrow} 0$.

First we choose $M \in \mathbb{N}$ such that $\pi\left(t_{\ell}-t_{m}\right) \leq \epsilon / C^{2}, \forall \ell, m \geq M$. Using (5.2) we have

$$
\pi\left(R_{n}\left(t_{m}-t_{\ell}\right)\right) \leq \epsilon, \forall \ell, m \geq M, \forall n \in \mathbb{N} .
$$

We now fix $n \in \mathbb{N}$ and take $m \geq M$. Equality (5.4) shows that the set $\left\{f_{j} \otimes g_{k}\right.$ : $1 \leq j, k \leq n\}$ is a basis for the space $R_{n}\left(X_{1} \otimes X_{2}\right)$. We consider on this space the norm

$$
\|t\|=\max \left\{\left|a_{j, k}\right|: t=\sum_{1 \leq j, k \leq n} a_{j, k} f_{j} \otimes g_{k}\right\} .
$$

Since $\operatorname{dim} R_{n}\left(X_{1} \otimes X_{2}\right)<\infty$, then $\|\cdot\|$ and $\pi$ are equivalent norms in $R_{n}\left(X_{1} \otimes X_{2}\right)$. From the hypotheses of the Lemma and from (5.4) it follows that $\left\|R_{n}\left(t_{\ell}\right)\right\| \rightarrow 0$. Hence we also have $\pi\left(R_{n}\left(t_{\ell}\right)\right) \rightarrow 0$. So after letting $\ell \rightarrow \infty$, from (5.6) we obtain for a fixed $\epsilon$ a natural number $M$ such that

$$
\pi\left(R_{n}\left(t_{m}\right)\right) \leq \epsilon, \forall m \geq M, \forall n \in \mathbb{N} .
$$

We now fix $m \geq M$ and let $n \rightarrow \infty$ in the above inequality. Using (5.3) we have $\pi\left(t_{m}\right) \leq \epsilon, \forall m \geq M$. This establishes that $t_{m} \stackrel{\pi}{\rightarrow} 0$.

Theorem 5.2. For $1<p<\infty$ the space $L^{p}\left(\mu_{1}\right) \otimes_{\pi} L^{p}\left(\mu_{2}\right)$ is $L^{0}\left(\mu_{1} \times \mu_{2}\right)$ completable, that is, $L^{p}\left(\mu_{1}\right) \widehat{\otimes}_{\pi} L^{p}\left(\mu_{2}\right)$ is continuously included in $L^{0}\left(\mu_{1} \times \mu_{2}\right)$.

Proof. First we notice that Proposition 4.5 implies that $L^{p}\left(\mu_{1}\right) \otimes_{\pi} L^{p}\left(\mu_{2}\right)$ has the $\mu_{1} \otimes \mu_{2}$-subsequence property, that is, a) in Definition 3.8.

To obtain the conclusion we will now show that the requirements of Lemma 5.1 holds for the particular choice of the present result. So consider $\varphi \in L^{p}\left(\Omega_{1}\right)^{*}$ and 
$\psi \in L^{p}\left(\Omega_{2}\right)^{*}$. Let $s>1$ be the conjugated exponent for $p$ and take $h_{j} \in L^{s}\left(\mu_{j}\right), j=$ 1,2 , such that

$$
\varphi(f)=\int_{\Omega_{1}} h_{1} f d \mu_{1}, \forall f \in L^{p}\left(\mu_{1}\right) ; \quad \psi(g)=\int_{\Omega_{2}} h_{2} g d \mu_{2}, \forall g \in L^{p}\left(\mu_{2}\right) .
$$

Then for $f \in L^{p}\left(\mu_{1}\right)$ and $g \in L^{p}\left(\mu_{2}\right)$ we have

$$
\begin{aligned}
\varphi \otimes \psi(f \otimes g) & =\varphi(f) \psi(g)=\left(\int_{\Omega_{1}} h_{1} f d \mu_{1}\right)\left(\int_{\Omega_{2}} h_{2} g d \mu_{2}\right) \\
& =\int_{\Omega_{1} \times \Omega_{2}}\left(h_{1} f\right) \otimes\left(h_{2} g\right) d \mu_{1} \otimes \mu_{2} \\
& =\int_{\Omega_{1} \times \Omega_{2}} h_{1} \otimes h_{2} \cdot f \otimes g d \mu_{1} \otimes \mu_{2} .
\end{aligned}
$$

Hence

$$
\varphi \otimes \psi(t)=\int_{\Omega_{1} \times \Omega_{2}} h_{1} \otimes h_{2} \cdot t d \mu_{1} \otimes \mu_{2}, \forall t \in L^{p}\left(\mu_{1}\right) \otimes L^{p}\left(\mu_{2}\right) .
$$

Consider now a bounded sequence $\left\{t_{n}\right\}$ in $L^{p}\left(\mu_{1}\right) \otimes_{\pi} L^{p}\left(\mu_{2}\right)$ (and so in $L^{p}\left(\mu_{1} \otimes \mu_{2}\right)$ ), that converges to $0 \mu_{1} \otimes \mu_{2}$-a.e. Take $\alpha_{m}=h_{1} \otimes h_{2} \cdot t_{m}, \forall m \in \mathbb{N}$ and $D>0$ such that $\left\|t_{m}\right\|_{L^{p}\left(\mu_{1} \otimes \mu_{2}\right)} \leq D, \forall m \in \mathbb{N}$. By Proposition 4.7 we have $L^{p}\left(\mu_{1}\right) \otimes L^{p}\left(\mu_{2}\right) \subset$ $L^{p}\left(\mu_{1} \otimes \mu_{2}\right)$ and also $L^{s}\left(\mu_{1}\right) \otimes L^{s}\left(\mu_{2}\right) \subset L^{s}\left(\mu_{1} \otimes \mu_{2}\right)$. Therefore

$$
\begin{aligned}
\int_{A}\left|\alpha_{m}\right| d \mu_{1} \otimes \mu_{2} & \leq\left\|t_{m}\right\|_{L^{p}\left(\mu_{1} \otimes \mu_{2}\right)}\left\|\chi_{A} h_{1} \otimes h_{2}\right\|_{L^{s}\left(\mu_{1} \otimes \mu_{2}\right)} \\
& \leq D \quad\left\|\chi_{A} h_{1} \otimes h_{2}\right\|_{L^{s}\left(\mu_{1} \otimes \mu_{2}\right)}, \forall A \in \Sigma_{1} \otimes \Sigma_{2} .
\end{aligned}
$$

Since $s>1$, from the above inequality it follows that the sequence $\left\{\alpha_{m}\right\}$ is equiintegrable. We also have $\alpha_{m} \rightarrow 0, \mu_{1} \otimes \mu_{2}$-a.e.. Thus we can now apply Vitali's convergence theorem ([10, Thm. III.3.6]) to find that

$$
\varphi \otimes \psi\left(t_{m}\right)=\int_{\Omega_{1} \times \Omega_{2}} \alpha_{m} d \mu_{1} \otimes \mu_{2} \rightarrow 0 .
$$

An application of Lemma 5.1 gives b) of Definition 3.8, and so the result holds.

Banach sequence spaces with $\left\{e_{n}\right\}$ as a Schauder basis. Next we will specialize to $\Omega_{1}=\Omega_{2}=\mathbb{N}$ and consider a normed tensor product $X_{1}(\mathbb{N}) \otimes_{q} X_{2}(\mathbb{N})$, where $X_{1}=X_{1}(\mathbb{N})$ and $X_{2}=X_{2}(\mathbb{N})$ are Banach sequence spaces, each of them having the canonical basis $\left\{e_{n}: n \in \mathbb{N}\right\}$ as a Schauder basis. By $q$ we denote a uniform reasonable crossnorm on $X_{1} \otimes X_{2}$. So now we have $f_{j}=e_{j}=g_{j}, \forall j \in \mathbb{N}$. Let $\left\{f_{j}^{*}\right\}$ be the biorthogonal basic sequence of $\left\{e_{n}\right\}$ with respect to $X_{1}$ and let $\left\{g_{k}^{*}: k \in \mathbb{N}\right\}$ be the biorthogonal basic sequence of $\left\{e_{n}: n \in \mathbb{N}\right\}$ with respect to $X_{2}$. Notice that $f_{j}^{*}$ and $g_{k}^{*}$ are just the evaluation maps on $j$ and $k$, respectively. From (5.1) we have

$$
\left\|f_{j}^{*}\right\| \leq C, \quad\left\|g_{j}^{*}\right\| \leq C, \forall j \in \mathbb{N} .
$$

Take $f=\left(f_{\ell}\right) \in X_{1}, g=\left(g_{\ell}\right) \in X_{2}$. Then $f_{j}^{*} \otimes g_{k}^{*}(f \otimes g)=f_{j}^{*}(f) g_{k}^{*}(g)=f_{j} g_{k}=$ $f \otimes g(j, k)$. Hence 


$$
f_{j}^{*} \otimes g_{k}^{*}(t)=t(j, k), \forall t \in X_{1} \otimes X_{2}, \forall j, k \in \mathbb{N} .
$$

Thus

$$
|t(j, k)| \leq\left\|f_{j}^{*}\right\|\left\|g_{k}^{*}\right\|\|t\|_{q} \leq 4 C^{2}\|t\|_{q} .
$$

Theorem 5.3. Let $X_{1}=X_{1}(\mathbb{N})$ and $X_{2}=X_{2}(\mathbb{N})$ be Banach sequence spaces with $\left\{e_{n}: n \in \mathbb{N}\right\}$ as a Schauder basis. If $q$ is a uniform and reasonable crossnorm on $X_{1} \otimes X_{2}$, then the normed space $X_{1} \otimes_{q} X_{2} \subset F\left(\mathbb{N}^{2}\right)$ is F-completable.

Proof. Let $\left\{t_{m}\right\} \subset X_{1} \otimes_{q} X_{2}$ be such that $t_{m} \stackrel{q}{\rightarrow} 0$. Then from (5.10) follows that $t_{m}(j, k)=f_{j}^{*} \otimes g_{k}^{*}\left(t_{m}\right) \rightarrow 0, \forall j, k \in \mathbb{N}$. This shows that $X_{1} \otimes_{q} X_{2}$ has the subsequence property and also that Lemma 5.1 applies.

Under the conditions of the above theorem, note that (5.10) implies that in fact

$$
X_{1} \widehat{\otimes}_{q} X_{2} \subset \ell^{\infty}\left(\mathbb{N}^{2}\right) .
$$

\section{Applichtions: an optimal faCtorization norm}

The optimal domain of a linear operator acting on an order continuous Banach function space was firstly represented as a concrete space of vector measure integrable functions by Curbera and Ricker (see [7, 8, 18] and the references therein). The original technique is strongly based on the lattice structure of the domain space. Thus, as a consequence of the lack of lattice structure of the normed tensor products analyzed in this paper, there is a deep methodological problem when the same approach is attempted in the case of bilinear maps. In this section we show how we can apply the results obtained in the rest of the paper to establish optimal factorizations for this case.

Let $\left(\Omega_{j}, \Sigma_{j}, \mu_{j}\right)$ be a $\sigma$-finite measure space, $j=1,2$. In the following we will consider $E$ to be a Banach space, $Z=Z\left(\mu_{1} \otimes \mu_{2}\right)$ a 2-rectangular normed space with respect to a semialgebra $\mathcal{S}$ contained in $\Sigma_{1} \otimes \Sigma_{2}$ and $T: Z \rightarrow E$ a bounded linear map. Clearly by taking $j$ as the identity and $Z_{T}=Z$ the next diagram holds:

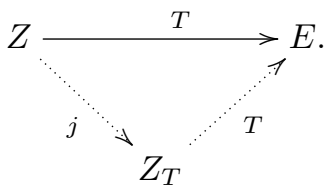

We are now interested in analyzing if there is some "minimal" 2-rectangular norm on $Z$-associated to a "maximal" space-, say $\|\cdot\|_{T}$, such that the operator $T:\left(Z,\|\cdot\|_{T}\right) \rightarrow E$ is still continuous.

Let $f \in Z$ and $R \in \mathcal{S}$. Then

$$
\left\|T\left(\chi_{R} f\right)\right\| \leq\|T\|\left\|\chi_{R} f\right\|_{Z} \leq C\|T\|\|f\|_{Z},
$$

where $C>0$ is a 2-rectangularity constant for $Z$. This allows us to define

$$
\|f\|_{T}=\sup \left\{\left\|T\left(\chi_{R} f\right)\right\|: R \in \mathcal{S}\right\}, \quad f \in Z .
$$


It is rapidly checked that $\|\cdot\|_{T}$ defines a seminorm on $Z$. As can be easily seen, for example by taking $T=0$, this seminorm may not be a norm. To distinguish those linear operators for which it is a norm we introduce the following definition. It can be seen as a kind of extension to our setting of the well-known notion of $\mu$-determined operator — see Section 2 and [18]-.

Definition 6.1. A bounded linear map $T: Z \rightarrow E$ is said to be 2-rectangularly determined, if the seminorm $\|\cdot\|_{T}$ is a norm. In this case we define $Z_{T}:=\left(Z,\|\cdot\|_{T}\right)$.

From (6.2) we have that the inclusion $j: Z \rightarrow Z_{T}$ is continuous:

$$
\|f\|_{T} \leq C\|T\|\|f\|_{Z},
$$

The following is our optimal norm result.

Theorem 6.2. Let $Z=Z\left(\mu_{1} \otimes \mu_{2}\right)$ be a 2-rectangular n.s. and $T: Z \rightarrow E a$ 2-rectangularly determined linear operator. Then:

i) $Z_{T}$ is also a 2-rectangular normed space.

ii) $T_{E}:=T: Z_{T} \rightarrow E$ is bounded.

iii) If $\|\cdot\|$ is any 2-rectangular norm on the vector space $Z$ and for $W=(Z,\|\cdot\|)$ the linear operator $T_{W}=T: W \rightarrow E$ is bounded, then the inclusion $j: W \rightarrow Z_{T}$ is continuous.

iv) If $W$ is dense in $Z$, then $W$ is also dense in $Z_{T}$.

Proof. i) Take $f \in Z_{T}$ and consider any $S \in \mathcal{S}$. Note that $\chi_{S} \cdot \chi_{R}=\chi_{S \cap R}, \forall R \in \mathcal{S}$. Hence

$$
\left\|\chi_{S} f\right\|_{T}=\sup _{R \in \mathcal{S}}\left\|T\left(\chi_{R} \chi_{S} f\right)\right\|=\sup _{R \in \mathcal{S}}\left\|T\left(\chi_{R \cap S} f\right)\right\| \leq\|f\|_{T} .
$$

ii) Take $f \in Z_{T}$. Since $\Omega \in \mathcal{S}$ we have $\|T f\|_{E}=\left\|T\left(\chi_{\Omega} f\right)\right\|_{E} \leq\|f\|_{T}$. This shows the linear operator $T_{E}$ is bounded and $\left\|T_{E}\right\| \leq 1$.

iii) Assume that a normed space $W=(Z,\|\cdot\|)$ is 2-rectangular and the linear operator $T: W \rightarrow E$ is bounded. Let $C$ be a 2-rectangularity constant for $W$. Take $f \in W$ and $R \in \mathcal{S}$. Then

$$
\left\|T\left(\chi_{R} f\right)\right\|_{E}=\left\|T_{W}\left(\chi_{R} f\right)\right\|_{E} \leq\left\|T_{W} \mid\right\| \chi_{R} f\left\|_{W} \leq C\right\| T_{W}\|\| f \|_{W} .
$$

Hence $\|f\|_{T} \leq C\left\|T_{W}\right\|\|f\|_{W}$.

iv) Since the inclusion $j: Z \rightarrow Z_{T}$ is continuous, the density of $W$ in $Z$, implies that of $W$ in $Z_{T}$.

Let us suppose additionally that the normed space $Z_{T}$ is $L^{0}(\mu)$-completable. Since $T: Z_{T} \rightarrow E$ is bounded let us consider its bounded linear extension $\widehat{T}$ : $\widehat{Z_{T}} \rightarrow E$. By Lemma $4.2 \widehat{Z_{T}}$ is also 2-rectangular. Hence it is a 2-rectangular Banach space with $Z=Z_{T}$ as a dense subspace and having a bounded linear extension of $T: Z \rightarrow E$. In this setting we have the following Optimal Domain Theorem.

Theorem 6.3. Let $Z=Z\left(\mu_{1} \otimes \mu_{2}\right)$ be a 2-rectangular normed space, $E$ a Banach space and $T: Z \rightarrow E$ a bounded linear operator. Assume also that $T$ is 2-rectangularly determined and that $Z_{T}$ is $L^{0}(\mu)$-completable. 
If $W=W\left(\mu_{1} \times \mu_{2}\right)$ is a 2-rectangular normed space having $Z$ as a dense subspace and there is a bounded linear map $T_{W}: W \rightarrow E$ with $T_{W}=T$ on $Z$, then $W$ is continuously included in $\widehat{Z_{T}}$ and $\widehat{T}$ extends $T_{W}$.

Proof. Let $C$ be a 2-rectangularity constant for $W$. Take $f \in Z$ and $R \in \mathcal{S}$. Then $\left\|T \chi_{R} f\right\|_{E}=\left\|T_{W} \chi_{R} f\right\|_{E} \leq\left\|T_{W}\right\|\left\|\chi_{R} f\right\|_{W} \leq C\left\|T_{W}\right\|\|f\|_{W}$. Hence

$$
\|f\|_{T} \leq C\left\|T_{W}\right\|\|f\|_{W}, \forall f \in Z .
$$

Take $f \in W$ and let $\left\{f_{n}\right\} \subset Z$ be a sequence satisfying $f_{n} \stackrel{W}{\rightarrow} f$. It follows now from (6.4) that $\left\{f_{n}\right\}$ is a Cauchy sequence in $Z_{T}$. Hence there is some $g \in \widehat{Z_{T}}$ such that $\left\|f_{n}-g\right\|_{\widehat{Z_{T}}} \rightarrow 0$. By Proposition 3.6 this implies that there is some subsequence $\left\{f_{n(k)}\right\}$ that converges pointwise $\mu$-a.e. to $g$. On the other hand, we also have that $f_{n} \rightarrow f$ pointwise $\mu$-a.e. Hence $f=g \in \widehat{Z_{T}}$ and (6.4) implies that

$$
\|f\|_{\widehat{Z_{T}}}=\lim _{n \rightarrow \infty}\left\|f_{n}\right\|_{T} \leq C\left\|T_{W}\right\|\|f\|_{W} .
$$

This establishes that $W$ is continuously included in $\widehat{Z_{T}}$. Moreover, we have $T_{W} f=\lim _{n \rightarrow \infty} T_{W}\left(f_{n}\right)=\lim _{n \rightarrow \infty} T\left(f_{n}\right)=\widehat{T}(f)$.

To end this work, we discuss the question of $T$ being 2-rectangularly determined in the case $\Omega_{1}=\Omega_{2}=\mathbb{N}$ and $\Sigma_{1}=\Sigma_{2}=\mathbb{N}$.

Theorem 6.4. Let $Z=Z\left(\mathbb{N}^{2}\right)$ be a 2-rectangular normed space and $T: Z \rightarrow E$ a bounded linear operator. If $w \in A=\left\{x \in \mathbb{N}^{2}: f(x) \neq 0\right.$ for some $\left.f \in Z\right\}$, then $\chi_{\{w\}} \in Z$ and $T$ is 2-rectangularly determined if, and only if, $T\left(\chi_{\{w\}}\right) \neq 0, \forall w \in A$.

Proof. If $w \in A$, then we have that $\chi_{\{w\}} \in Z$. Assume first that $T$ is 2-rectangularly determined. Take $w \in A$. Then

$$
0 \neq\left\|\chi_{\{w\}}\right\|_{T}=\sup \left\{\left\|T\left(\chi_{R} \chi_{\{w\}}\right)\right\|_{E}: R \in \mathcal{S}\right\}=\left\|T\left(\chi_{\{w\}}\right)\right\|
$$

Assume now that $T\left(\chi_{\{w\}}\right) \neq 0, \forall w \in A$. Take $f \in Z, f \neq 0$ and fix $w \in \mathbb{N}^{2}$ such that $f(w) \neq 0$. Then $\chi_{\{w\}} f=f(w) \chi_{\{w\}}$. So $T\left(\chi_{\{w\}} f\right)=f(w) T\left(\chi_{\{w\}}\right) \neq 0$.

\section{REFERENCES}

[1] Y. A. Abramovich and C. D. Aliprantis, An invitation to operator theory. Graduate Studies in Mathematics, Vol 50, AMS, 2002.

[2] C. Bennett and R. Sharpley, Interpolation of operators, Academic Press, Boston, 1988.

[3] Q. Bu, G. Buskes, and A.G. Kusraev, 'Bilinear maps on products of vector lattices: A survey' in Positivity-Trends in Mathematics, Ed. K. Boulabiar, G. Buskes and A. Triki, Birkhäser Verlag AG, Basel, 2007, 97-126.

[4] G. Buskes, and A. Van Rooij, 'Bounded variation and tensor products of Banach lattices', Positivity 2003, 47-59.

[5] J.M. Calabuig, M. Fernández-Unzueta, F. Galaz-Fontes, F., and E.A. Sánchez-Pérez, 'Extending and factorizing bounded bilinear maps defined on order continuous Banach function spaces', RACSAM 2014, 353-367.

[6] J.M. Calabuig, M. Fernández-Unzueta, F. Galaz-Fontes, and E.A. Sánchez-Pérez, 'Equivalent norms in a Banach function space and the subsequence property'. Submitted for publication.

[7] G.P. Curbera, and W.J. Ricker, 'Optimal domains for kernel operators via interpolation', Math. Nachr. 2002, 47-63. 
[8] G.P. Curbera, and W.J. Ricker, 'Vector measures, integration and applications', in: Positivity. Birkhäuser Basel, 2007, 127-160.

[9] J.G. de Lamadrid, 'Uniform cross norms and tensor products', Duke Math. J. 1965, 797-803.

[10] N. Dunford and J. Schwartz, Linear operators, Part I: General theory, Interscience Publishers Inc., New York, 1958.

[11] D.H. Fremlin, 'Tensor products of Archimedean vector lattices', Amer J. Math. 1972, 777-798.

[12] D.H. Fremlin, 'Tensor products of Banach lattices', Math. Ann. 1974, 87-106.

[13] K.L. Yew, 'Completely p-summing maps on the operator Hilbert space OH', Journal of Functional Analysis 255 (2008), 1362-1402.

[14] S. Kwapien and A. Pelczynski, 'The main triangle projection in matrix spaces and its applications', Studia Math. 1970, 43-68.

[15] J. Lindenstrauss and L. Tzafriri, Classical Banach spaces II, Springer Verlag, Berlin, 1979.

[16] W.A.J. Luxemburg and A.C. Zaanen, Riesz Spaces I, Amsterdam, North-Holland Publishing Company, 1971.

[17] M. Milman, Some new function spaces and their tensor products, Depto. de Matemática, Facultad de Ciencias, U. de los Andes, Mérida, Venezuela, 1978.

[18] S. Okada, W.J. Ricker and E.A. Sánchez Pérez, Optimal Domain and Integral Extension of Operators acting in Function Spaces. Oper. Theory Adv. Appl., vol. 180, Birkhäuser, Basel, 2008.

[19] A.R. Schep, 'Factorization of positive multilinear maps', Illinois J. Math. 1984, 579-591.

[20] A.C. Zaanen, Integration. Amsterdam-New York, North-Holland Publishing Company, 1967.

[21] A.C. Zaanen, Riesz Spaces II, Amsterdam, North-Holland Publishing Company, 1983.

Instituto Universitario de Matemática Pura y Aplicada, Universitat Politècnica de València, Camino de Vera S/n, 46022 Valencia, Spain

E-mail address: jmcalabu@mat.upv.es

Centro de Investigación em Matemáticas, A.C., Jalisco S/N, Col. Valenciana CP: 36240 Guanajuato, Gto., México

E-mail address: maite@cimat.mx

Centro de Investigación en Matemáticas, A.C., Jalisco S/N, Col. Valenciana CP: 36240 Guanajuato, Gto., México

E-mail address: galaz@cimat.mx

Instituto Universitario de Matemática Pura y Aplicada, Universitat Politècnica de València, Camino de Vera s/n, 46022 Valencia, Spain

E-mail address: easancpe@mat.upv.es 